From the Department of Pediatrics, University of South Florida and the Health Outcomes and Behavior Program, H. Lee Moffitt Cancer Center and Research Institute, Tampa, FL.

Submitted August 30, 2006; accepted July 20, 2007; published online ahead of print at www.jco.org on September 17, 2007

Supported by National Cancer Institute Grant No. CA81920.

Authors' disclosures of potential conflicts of interest and author contributions are found at the end of this article.

Address reprint requests to Paul $\mathrm{B}$. Jacobsen, PhD, H. Lee Moffitt Cancer Center, 12902 Magnolia Dr, MRC-PSY, Tampa, FL 33612; e-mail: paul.jacobsen@ moffitt.org.

() 2007 by American Society of Clinical Oncology

0732-183X/07/2529-4657/\$20.00

DOI: 10.1200/JCO.2006.09.0126

\title{
Self-Administered Stress Management Training in Patients Undergoing Radiotherapy
}

Mindy M. Krischer, Ping Xu, Cathy D. Meade, and Paul B. Jacobsen

$$
\text { A } \begin{array}{llllllll}
\text { B } & \text { S } & \text { T } & \text { R } & \text { A } & \text { C } & \text { T }
\end{array}
$$

\section{Purpose}

This study sought to continue research on psychosocial interventions for patients being treated with radiation therapy across multiple centers and to replicate positive findings of a single-center study of patients being treated with chemotherapy. The primary objective of this study was to determine if a stress management intervention was effective in improving quality of life and decreasing psychological distress in patients undergoing radiotherapy for cancer.

\section{Patients and Methods}

A total of 310 patients about to begin radiotherapy treatment were randomly assigned to receive usual care only or self-administered stress management training. Quality-of-life assessments occurred at baseline and for 3 weeks after the beginning of radiotherapy treatment.

\section{Results}

Overall, patients assigned to receive stress management training did not report significantly less psychological distress on the Medical Outcomes Study 36-Item Short Form (SF-36) Mental Component Summary Scale than did those assigned to usual care. When divided into subgroups based on the SF-36 Mental Component Summary Scale scores immediately after their first radiotherapy treatment, patients with above-average levels of psychological distress (scores $\leq 50$ ) who were randomly assigned to the intervention condition reported significant improvement in their distress compared with those assigned to usual care only on the SF-36 Mental Health Subscale and the Center for Epidemiologic Studies Depression Scale.

\section{Conclusion}

This study found that self-administered stress management training is effective only in those radiotherapy patients with initially higher levels of psychological distress. Additional research should examine the benefits of stress management training targeted specifically to patients experiencing heightened distress.

\section{J Clin Oncol 25:4657-4662. (C) 2007 by American Society of Clinical Oncology}

\section{INTRODUCTION}

Radiotherapy is one of the most effective means of controlling local/regional cancer. As such, the adverse effects of the treatment have been well documented. In addition to common adverse effects such as fatigue, many patients experience heightened emotional distress as a consequence of receiving radiotherapy. ${ }^{1-4} \mathrm{~A}$ number of randomized clinical trials have documented the benefits of psychosocial interventions for patients undergoing radiotherapy. In one trial involving patients undergoing curative and palliative radiotherapy, patients receiving professionally administered relaxation training with guided imagery experienced significant reductions in depression and fatigue relative to a no-treatment control group. ${ }^{5}$ Other trials have documented the benefits of group psychotherapy ${ }^{6}$ and a nursing in- tervention ${ }^{7}$ on emotional and physical distress in cancer patients undergoing radiotherapy.

Because of the costly nature of providing professionally administered psychosocial interventions, research is needed that focuses on producing more accessible interventions that would be just as effective. A previous study by Jacobsen et $\mathrm{al}^{8}$ evaluated the efficacy and economic costs of a patient selfadministered form of stress management training for patients undergoing chemotherapy. Three hundred eighty-two cancer patients about to begin chemotherapy treatment were randomly assigned to one of three intervention conditions: usual care only (UCO), clinician-administered stress management training (CSMT), or self-administered stress management training (SSMT). In the CSMT condition, patients received professionally administered training in progressive muscle relaxation and guided 
imagery, abdominal breathing, and use of coping self-statements from a psychologist during a 60-minute session before the start of treatment. In the SSMT condition, patients were provided with a videocassette, audiocassette, and brochure that provided directions in the use of the same three stress management techniques. Outcome measures (Medical Outcomes Study 36-Item Short Form [SF-36] Mental Component Summary Scale, Center for Epidemiologic Studies Depression Scale [CES-D], and Spielberger State-Trait Anxiety Inventory [STAIS]) were assessed at baseline (before receipt of the interventions) and just before the start of the second, third, and fourth chemotherapy cycles. Compared with patients who received UCO, patients receiving SSMT reported significantly $(P<.05)$ better physical functioning, greater vitality, fewer role limitations due to emotional problems, and better mental health. In contrast, patients who received CSMT fared no better in terms of quality of life than patients receiving UCO.

This study sought to continue research on psychosocial interventions by replicating the single-center study conducted by Jacobsen et $\mathrm{al}^{8}$ in patients being treated with radiotherapy in multiple centers. In doing so, this study used much of the same research methodology and instruments. Because of the absence of benefits for CSMT in the original study, patients were randomly assigned to receive either SSMT in addition to the patient's usual psychosocial care or UCO. The primary objective of this study was to determine if a modified version of this stress management intervention was effective in improving quality of life and decreasing psychological distress (anxiety and depression) in patients undergoing radiotherapy for cancer.

\section{PATIENTS AND METHODS}

\section{Participants}

To be eligible, patients had to be at least 18 years of age, diagnosed with cancer, scheduled to receive a minimum of 12 radiotherapy treatments during a 21-day period, be capable of reading and speaking English, and be able and willing to provide written informed consent before study entry.

A total of 327 patients provided informed consent and were randomly assigned to the intervention condition. After random assignment, five participants were declared ineligible (treatment plan changes) and eight withdrew before the protocol intervention. Three participants withdrew during the protocol and one patient was lost to follow-up. The remaining 310 patients (UCO, $\mathrm{n}=156$; SSMT, $\mathrm{n}=154$ ) completed at least one of the assessments (week 1, n = 302; week 2, $\mathrm{n}=300$; week $3, \mathrm{n}=302$ ) and were included in the outcome analyses described.

The mean age of participants was 60.96 years (standard deviation, 12.25 years; Table 1$)$. The majority were female $(71.6 \%)$, white $(91.6 \%)$, and currently married (69\%). Slightly more than half had attended college $(54.8 \%)$. The median household income was in the $\$ 40,000$ to $\$ 74,000 /$ year range. Self-reported performance status was characterized by no physical problems (44\%), minor physical problems (35.2\%), or significant physical problems (10.9\%).

The baseline characteristics of individuals assigned to each treatment condition were analyzed using $\chi^{2}$ tests for categoric variables and $t$ tests for continuous variables. These analyses showed no significant $(P \leq .05)$ differences in the patient characteristics between the two treatment arms.

\section{Procedure}

Patients were recruited by clinical trials coordinators from 21 sites associated with the Community Clinical Oncology Program (a large network that enables patients and physicians to participate in clinical trials sponsored by the National Cancer Institute). After providing informed consent, patients completed a baseline assessment and were randomly assigned to one of the two study conditions. Randomization was conducted using an automated interac-

\begin{tabular}{|c|c|c|}
\hline \multicolumn{3}{|c|}{$\begin{array}{c}\text { Table 1. Participant Demographics and Clinical Characteristics by } \\
\text { Treatment Arm }\end{array}$} \\
\hline Characteristic & $\begin{array}{l}\text { Stress } \\
\text { Management }\end{array}$ & $\begin{array}{l}\text { Usual Care } \\
\text { Only }\end{array}$ \\
\hline No. of participants & 154 & 156 \\
\hline Average age, years & 60.3 & 61.6 \\
\hline \multicolumn{3}{|l|}{ Sex } \\
\hline Female & 110 & 112 \\
\hline Male & 44 & 44 \\
\hline \multicolumn{3}{|l|}{ Race/ethnicity } \\
\hline White & 142 & 142 \\
\hline African American & 9 & 8 \\
\hline American Indian or Alaskan Native & 1 & 4 \\
\hline Asian & 2 & 1 \\
\hline Native Hawaiian or other Pacific Islander & 0 & 1 \\
\hline \multicolumn{3}{|l|}{ Marital status } \\
\hline Married & 103 & 111 \\
\hline Divorced & 29 & 21 \\
\hline Separated & 3 & 1 \\
\hline Widowed & 11 & 13 \\
\hline Single, never married & 7 & 8 \\
\hline \multicolumn{3}{|l|}{ Highest level of education achieved } \\
\hline Unknown & 2 & 0 \\
\hline 8 th grade or less & 3 & 7 \\
\hline Some high school (grades 9-12) & 9 & 18 \\
\hline High school graduate or GED & 59 & 42 \\
\hline Vocational school or some college & 46 & 43 \\
\hline College or university graduate & 21 & 24 \\
\hline Professional or graduate school experience & 14 & 22 \\
\hline \multicolumn{3}{|l|}{ Household annual income range } \\
\hline Unknown & 2 & 3 \\
\hline Less than $\$ 10,000$ & 12 & 10 \\
\hline$\$ 10,000-\$ 19,999$ & 20 & 20 \\
\hline$\$ 20,000-\$ 39,999$ & 43 & 33 \\
\hline$\$ 40,000-\$ 74,999$ & 37 & 43 \\
\hline$\$ 75,000$ or more & 19 & 17 \\
\hline Prefer not to answer & 21 & 30 \\
\hline \multicolumn{3}{|l|}{ Cancer type } \\
\hline Breast cancer & 88 & 100 \\
\hline Prostate cancer & 33 & 32 \\
\hline Lung cancer & 4 & 9 \\
\hline Head and neck cancer & 10 & 3 \\
\hline Other & 15 & 8 \\
\hline Lymphoma & 4 & 4 \\
\hline \multicolumn{3}{|l|}{ Performance status } \\
\hline No physical problems & 61 & 75 \\
\hline Minor physical problems & 56 & 53 \\
\hline Significant physical problems & 35 & 28 \\
\hline Unknown & 2 & 0 \\
\hline
\end{tabular}

Abbreviation: GED, general equivalency diploma.

tive voice response system. Follow-up assessments occurred at 1, 2, and 3 weeks after the initiation of radiotherapy. These follow-up assessments were completed by the patient via the Internet or by mail.

UCO. All participants received the usual psychosocial care typically provided at the institution where they were receiving treatment. Each participant completed a usual care survey assessing their use of a support group, psychosocial counseling, community agencies, psychiatry, family conference and counseling, and pastoral counseling. Less than 10\% reported usage of any of these for emotional support. There was no significant difference in the usual care received by those in the UCO and SSMT arms.

SSMT. In addition to receiving usual psychosocial care, participants met individually with a clinician (typically a nurse) for approximately 5 min- 
utes. During this meeting, instructional materials were distributed and the patient was given a standardized explanation of the nature and purpose of the intervention. The instructional materials consisted of a 15-minute prerecorded videotape and a 12-page booklet modified for patients receiving radiation from the materials used in the previous study. ${ }^{8}$ In addition, participants received a 35-minute prerecorded audiotape entitled Active Relaxation (Leo Media Inc, Urbana, IL). The videotape and booklet presented the sources and manifestations of stress during radiotherapy and the potential benefits of stress management training. Also included was instruction on paced breathing, active relaxation, and positive thinking. Participants were instructed to view the videotape and then follow directions in the booklet. Both the video and booklet contained testimonials about the benefits of using each stress management technique, aimed at making the intervention credible to participants. The paced breathing exercise followed the format developed by Turk et al. ${ }^{9}$ The active relaxation exercise was similar to one developed by Burish et al. ${ }^{10}$ It combined abbreviated progressive muscle relaxation training with the use of relaxing mental imagery. Patients were directed to repeatedly tense and relax a standard set of muscle groups, after which they were assisted in visualizing a tranquil nature scene to enhance and sustain feelings of relaxation. The positive thinking exercise provided participants with brief instruction in the use of coping self-statements borrowed from stress inoculation training. ${ }^{11}$ Specifically, they were instructed to stop when experiencing symptoms of stress, think about negative things they might be saying to themselves about their ability to cope, and focus instead on more positive thoughts about coping with stress. As part of this exercise, participants were encouraged to identify specific coping self-statements they might use during radiotherapy treatment.

\section{Measures}

The primary outcome measure was the Mental Component Summary score of the Medical Outcomes Study SF-36. ${ }^{12}$ The SF-36 survey measures physical functioning, role limitations from physical or emotional conditions, bodily pain, general health perceptions, vitality, social functioning, and mental health. Combined, these measurements produce both a physical health and a mental health summary score. Scores on the SF-36 Mental Health Subscale were also examined as an outcome. Patients also completed the CES-D,${ }^{13}$ which measures depressive symptoms during the last week, and the State Version of the STAI-S, ${ }^{14}$ which measures current level of anxiety. Each of these measurements was administered at baseline and weeks 1, 2, and 3. Given that they were considered secondary end points, no adjustment was made in the statistics for multiple comparisons, and the reader should be cautioned regarding the increased likelihood of serendipitous findings that may result.

\section{Data Analysis}

The main analyses were done to test the hypothesis that participants receiving SSMT would report better quality of life and less depression and anxiety than those receiving UCO. Analysis for repeated measures was conducted using SAS PROC MIXED ${ }^{15}$ (SAS Institute, Cary, NC) based on an unstructured covariance matrix model assumption. The models used for analysis included the baseline measures as covariates: the effects of treatment alone, time alone, the interaction of treatment with time, the quadratic effect of time, and the quadratic effect of treatment and time. Inclusion of quadratic terms was based on inspection of mean outcomes scores at each assessment point, which clearly showed nonlinear changes over time. Tests of significance were two-tailed and a probability $(P) \leq .05$ was considered to be statistically significant. Statistical analyses were performed with SAS, version 9 (SAS Institute).

\section{RESULTS}

The Mental Component Summary Scale of the SF-36, the primary quality-of-life outcome, did not show a significant treatment by time interaction effect $(P=.22$; Table 2$)$. However, there was a significant treatment by time interaction $(P=.05)$ effect for the Mental Health Index subscale. Patients who received SSMT showed greater improvement in mental health than patients who received UCO. The effect, however, was nonlinear in that the largest improvement occurred just after treatment initiation (week 1) and diminished thereafter (Table 3$)$. This interaction effect was not significant $(P=.13)$ when the quadratic term was excluded from the analysis.

Additional analyses were conducted to determine if there were interactions between treatment and week 1 Mental Component Summary Scale scores (the first data point after initiation of radiotherapy) that would justify conducting analyses in which participants were divided into high-and low-distress subgroups based on their scores on this measure. The rationale for this approach is evidence suggesting that patients with worse functioning benefit more from psychosocial interventions. ${ }^{16}$ Week 1 rather than baseline scores were used to capture distress that might be related to initiation of radiotherapy. Mental Component Summary Scale scores are expressed as $t$ scores with a mean of 50 and a standard deviation of 10 , based on US population norms reported by the scale developers. ${ }^{17}$ Accordingly, those participants with a score greater than 50 had lower than average levels of psychological distress and little limitation in usual activities due to emotional distress (low-distress group). Those with a score $\leq 50$ had above-average levels of psychological distress and limitations in usual activities as a result of emotional problems (high-distress group). Findings indicated that there were significant interactions $(P<.001)$

Table 2. SSMT Treatment Effect Over Time

\begin{tabular}{|c|c|c|c|c|c|c|c|c|c|c|}
\hline \multirow[b]{2}{*}{ Measurement } & \multicolumn{2}{|c|}{ Treatment } & \multicolumn{2}{|c|}{ Time } & \multicolumn{2}{|c|}{$\begin{array}{l}\text { Treatment } \times \\
\text { Time }\end{array}$} & \multicolumn{2}{|c|}{ Time $\times$ Time } & \multicolumn{2}{|c|}{$\begin{array}{l}\text { Treatment } \times \\
\text { Time } \times \text { Time }\end{array}$} \\
\hline & $t$ & $P$ & $t$ & $P$ & $t$ & $P$ & $t$ & $P$ & $t$ & $P$ \\
\hline Mental Health Summary Scale & 1.02 & .31 & 0.85 & .40 & -1.23 & .22 & -1.06 & .29 & 1.24 & .22 \\
\hline Mental Health Index & 2.07 & .04 & 1.52 & .13 & -1.95 & .05 & -1.30 & .20 & 1.70 & .09 \\
\hline \multicolumn{11}{|l|}{ CES-D } \\
\hline Anxiety & 0.03 & .98 & -0.26 & .79 & 0.55 & .58 & 0.31 & .76 & -0.61 & .54 \\
\hline
\end{tabular}

Abbreviations: SSMT, self-administered stress management training; SF-36, Medical Outcomes Study 36-Item Short Form; CES-D, Center for Epidemiologic Studies Depression Scale; STAI-S, Spielberger State-Trait Anxiety Inventory. 


\begin{tabular}{|c|c|c|c|c|c|c|c|c|c|c|c|c|c|c|c|c|}
\hline \multirow[b]{3}{*}{ Measure } & \multicolumn{4}{|c|}{ Baseline } & \multicolumn{4}{|c|}{ First Week } & \multicolumn{4}{|c|}{ Second Week } & \multicolumn{4}{|c|}{ Third Week } \\
\hline & \multicolumn{2}{|c|}{ SSMT } & \multicolumn{2}{|c|}{ UCO } & \multicolumn{2}{|c|}{ SSMT } & \multicolumn{2}{|c|}{ UCO } & \multicolumn{2}{|c|}{ SSMT } & \multicolumn{2}{|c|}{ UCO } & \multicolumn{2}{|c|}{ SSMT } & \multicolumn{2}{|c|}{ UCO } \\
\hline & $\begin{array}{l}\text { Mean } \\
\text { Score }\end{array}$ & SD & $\begin{array}{l}\text { Mean } \\
\text { Score }\end{array}$ & SD & $\begin{array}{l}\text { Mean } \\
\text { Score }\end{array}$ & SD & $\begin{array}{l}\text { Mean } \\
\text { Score }\end{array}$ & SD & $\begin{array}{l}\text { Mean } \\
\text { Score }\end{array}$ & SD & $\begin{array}{l}\text { Mean } \\
\text { Score }\end{array}$ & SD & $\begin{array}{l}\text { Mean } \\
\text { Score }\end{array}$ & SD & $\begin{array}{l}\text { Mean } \\
\text { Score }\end{array}$ & SD \\
\hline \multicolumn{17}{|l|}{ SF-36 } \\
\hline Mental Health Summary Scale & 49.74 & 11.26 & 51.02 & 10.03 & 52.48 & 9.52 & 52.85 & 10.16 & 51.54 & 9.85 & 52.68 & 10.07 & 51.39 & 10.93 & 51.86 & 10.93 \\
\hline Mental Health Index & 75.19 & 19.81 & 77.51 & 16.27 & 80.77 & 15.94 & 81.04 & 17.57 & 79.30 & 17.96 & 82.43 & 17.27 & 79.97 & 19.81 & 82.26 & 18.01 \\
\hline \multicolumn{17}{|l|}{ CES-D } \\
\hline Depressive Symptoms & 11.93 & 9.88 & 10.12 & 8.72 & 9.18 & 8.27 & 9.30 & 9.35 & 9.80 & 8.75 & 8.82 & 8.99 & 10.17 & 9.18 & 9.28 & 9.67 \\
\hline \multicolumn{17}{|l|}{ STAI-S } \\
\hline Anxiety & 34.79 & 12.74 & 33.98 & 11.11 & 32.22 & 10.97 & 30.48 & 10.86 & 32.57 & 11.56 & 30.56 & 11.24 & 32.19 & 12.31 & 30.57 & 11.22 \\
\hline
\end{tabular}

between treatment and week 1 levels of distress for Mental Component Summary Scale scores, SF-36 Mental Health Subscale scores, CES-D scores, and STAI-S scores.

Accordingly, analyses of treatment effects were conducted in groups defined by their week 1 Mental Component Summary Scale scores. Among patients in the high-distress group, these analyses revealed a marginally significant treatment effect $(P=.06)$ for the SF-36 Mental Component Summary Scale (Table 4). In addition, the Mental Health Subscale of the SF-36 showed significant effects of treatment $(P=.02)$ when compared with the UCO group. The CES-D scale showed significant positive treatment $(P=.004)$ effects as well. Results for the STAI-S were not statistically significant. The treatment effect for the CES-D but not the Mental Health Subscale remained significant $(P=.006)$ when the quadratic term was excluded from the analysis. Data analysis in the low-distress subgroup revealed no significant treatment effects for the Mental Component Summary Scale, the Mental Health Subscale, the CES-D, or the STAI-S.

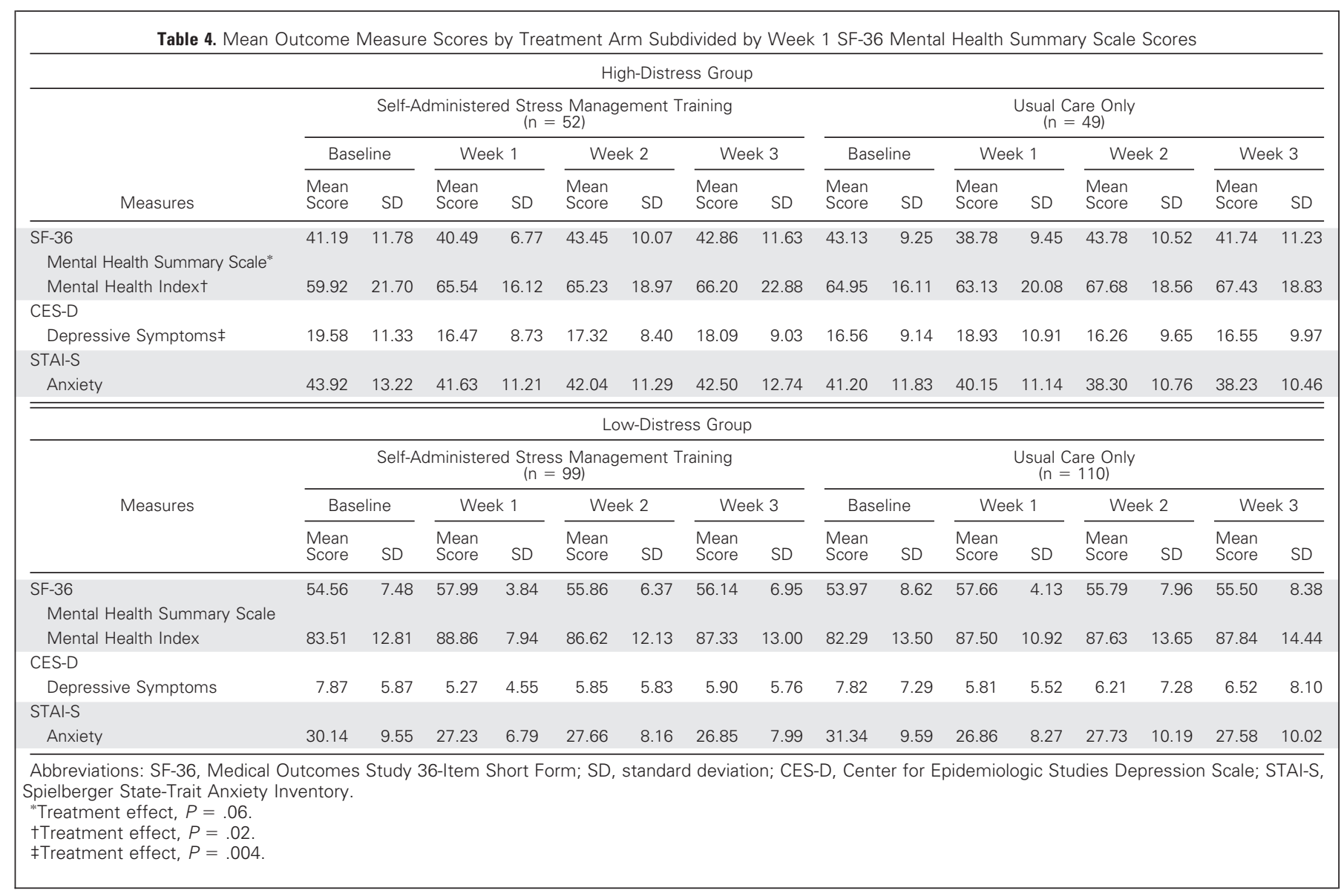


Estimates of effect sizes for the significant treatment effects observed in the high-distress group were determined by calculating the difference between the baseline values for each intervention arm and the average of values for weeks 1 to 3 for each intervention arm and dividing each of these change scores by the corresponding pooled standard deviation for the change in values. The resulting effect sizes were 1.19 for the Mental Component Summary Scale, 1.44 for the Mental Health Subscale, and 2.14 for the CES-D.

\section{DISCUSSION}

The lack of a significant effect for the SF-36 Mental Component Summary Scale indicates that SSMT did not significantly improve mental well-being relative to usual care. Moreover, the significant nonlinear effect of SSMT on SF-36 Mental Health Subscale scores suggests that the initial benefits of the intervention on mental health diminished over time. Therefore, the overall benefit of the intervention was limited.

The analysis of patient baseline characteristics found that patients began this study with close to an average level of mental health according to norms for the SF-36 Mental Component Summary Scale. This indicates that the average patient did not suffer from psychological distress that interfered with his or her daily life. However, standard deviations for the SF-36 Mental Component Summary Scale were relatively large, suggesting a large range of functionality at the beginning of the study.

Consequently, exploratory analyses were conducted in which the study population was divided into subgroups based on their week 1 SF-36 Mental Component Summary score to reflect their mental health after their first exposure to radiation therapy. Only in the subgroup with above-average levels of distress subsequent to radiation therapy was SSMT effective in promoting mental health and limiting depressive symptoms compared with UCO. These findings are consistent with research indicating that patients with initially worse functioning may benefit more from psychosocial interventions. ${ }^{16}$ The benefits of SSMT were mostly evident when quadratic terms were included in the analyses. Indeed, the sole effect of SSMT that remained significant when quadratic terms were excluded was the treatment effect on depressive symptoms in the high-distress group.

In contrast to the current study, a previous evaluation of SSMT with patients undergoing chemotherapy found that it significantly outperformed UCO on all four outcome measures used in the current study regardless of patients' initial level of distress. ${ }^{8}$ Differences in results between the two studies may reflect differences in the follow-up periods (three cycles of chemotherapy in the earlier study $v 3$ weeks of radiotherapy in the current study) and the standardization of usual care procedures in the earlier study but not the current study.

Several limitations of the current study should be noted. First, the subgroup analyses based on level of distress can only be considered exploratory, given that the subgroups were defined post hoc.
Future research should examine the benefits of SSMT targeted specifically to patients experiencing heightened distress. Second, the sample was relatively homogeneous in terms of race, education, and income. The effectiveness of this intervention strategy with a more diverse patient population needs to be addressed in future research. Third, the final study assessment occurred at end of the third week of radiotherapy. Whether the observed benefits of SSMT persist longer into the course of radiotherapy and perhaps into the period after completion of radiotherapy is not known. Finally, due to the method of recruiting, information was not collected about the number of patients who declined to participate or their characteristics. Thus, the acceptability of participating in this randomized controlled trial is not known. In addition, the possibility that the sample of participants differs from the larger group of patients asked to participate cannot be ruled out.

Although the principal analyses yielded negative results, exploratory analyses show that SSMT was effective in patients who were experiencing heightened psychological distress, allowing for more targeted approaches to behavioral intervention. As these analyses indicated, SSMT was effective in this group in multiple clinical settings with varying usual care programs. Differences on the Mental Component Summary Scale were only marginally significant; however, the Mental Health Subscale and the CES-D demonstrated larger differences. Nevertheless, all of the observed effect sizes (ie, improvements over time in the intervention group relative to the control group) were small, suggesting that the intervention had a modest impact on patient distress. The modest impact of the intervention must be weighed against the fact that, relative to professionally administered interventions, it can be more easily administered to patients and, therefore, has the potential to benefit more patients. In terms of its clinical application, study results suggest that implementation of the intervention should be reserved for patients experiencing heightened distress during radiotherapy, and that these patients should be monitored closely to determine if they require additional assistance in managing their distress.

AUTHORS' DISCLOSURES OF POTENTIAL CONFLICTS OF INTEREST

The author(s) indicated no potential conflicts of interest.

\section{AUTHOR CONTRIBUTIONS}

Conception and design: Cathy D. Meade, Paul B. Jacobsen

Data analysis and interpretation: Mindy M. Krischer, Ping Xu, Paul B. Jacobsen

Manuscript writing: Mindy M. Krischer, Ping Xu, Paul B. Jacobsen Final approval of manuscript: Mindy M. Krischer, Ping Xu, Cathy D. Meade, Paul B. Jacobsen

\section{REFERENCES}

1. Richardson $A$, Ream $E$ : The experience of fatigue and other symptoms in patients receiving chemotherapy. Eur J Cancer Care 5:24-30, 1996
2. Hickok JT, Morrow GR, McDonald S, et al: Frequency and correlates of fatigue in lung cancer patients receiving radiation therapy: Implications for management. J Pain Symptom Manage 11:370-377, 1996

3. Buick DL, Petrie KJ, Booth R, et al: Emotional and functional impact of radiotherapy and chemotherapy on patients with primary breast cancer. J Psychosoc Oncol 18:39-62, 2000

4. Smets EM, Visser MR, Willems-Groot AF, et al: Fatigue and radiotherapy: (A) Experience in patients undergoing treatment. $\mathrm{Br} \mathrm{J}$ Cancer 78:899906, 1998 
5. Decker TW, Cline-Elsen J, Gallagher M: Relaxation therapy as an adjunct in radiation oncology. $\mathrm{J}$ Clin Psychol 48:388-393,1992

6. Forester B, Kornfeld DS, Fleiss JL, et al: Group psychotherapy during radiotherapy: Effects on emotional and physical distress. Am J Psychiatry 150:1700-1706, 1993

7. Wengström $Y$, Haggmark $C$, Strander $H$, et al: Effects of a nursing intervention on subjective distress, side effects and quality of life of breast cancer patients receiving curative radiation therapy: A randomized study. Acta Oncol 38:763-770, 1999

8. Jacobsen PB, Meade CD, Stein KD, et al: Efficacy and costs of two forms of stress manage- ment training for cancer patients undergoing chemotherapy. J Clin Oncol 20:2851-2862, 2002

9. Turk DC, Meichenbaum D, Genest M Pain and Behavioral Medicine: A Cognitive Behavioral Perspective. New York, NY, Guilford Press, 1983

10. Burish TG, Snyder SL, Jenkins RA: Preparing patients for cancer chemotherapy: Effect of coping preparation and relaxation interventions. J Consult Clin Psychol 59:518-525, 1991

11. Meichenbaum D: Stress Inoculation Training New York, NY, Pergamon Press, 1985

12. Ware JE: SF-36 Health Survey. Manual and Interpretation Guide. Boston, MA, The Health Institute, New England Medical Center, 1993
13. Radloff LS: The CES-D Scale: A self-report depression scale for research in the general population. Appl Psychol Measure 1:385-401, 1977

14. Speilberger CD: Manual for the State-Trait Anxiety Inventory (Form Y). Palo Alto, CA, Consulting Psychologists Press, 1983

15. Little RC, Milliken GA, Stroup WW, et al: SAS System for Mixed Models. Cary, NC, SAS Institute, 1996

16. Sheard T, Maguire P: The effect of psychological interventions on anxiety and depression in cancer patients: Results of two meta-analyses. $\mathrm{Br} \mathrm{J}$ Cancer 80:1770-1780, 1999

17. Ware, JE: SF-36 Physical and Mental Health Summary Scales: A User's Manual. Boston, MA, The Health Institute, New England Medical Center, 1994

\section{Acknowledgment}

The Acknowledgment is included in the full-text version of this article, available online at www.jco.org. It is not included in the PDF version (via Adobe ${ }^{\circledR}$ Reader $^{\circledR}$ ). 